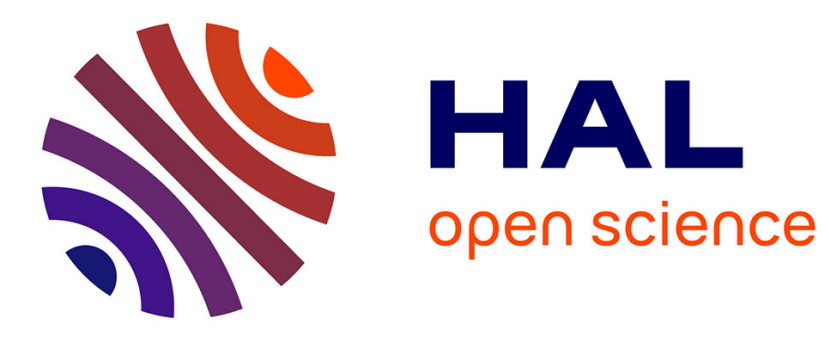

\title{
Experimental Measurement of Time Difference Of Arrival
}

\author{
Hugo Seuté, Cyrille Enderli, Jean-François Grandin, Ali Khenchaf, \\ Jean-Christophe Cexus
}

\section{- To cite this version:}

Hugo Seuté, Cyrille Enderli, Jean-François Grandin, Ali Khenchaf, Jean-Christophe Cexus. Experimental Measurement of Time Difference Of Arrival. International Radar Symposium 2016 - IRS2016, May 2016, Krakow, Poland. 4 p. hal-01326376

\section{HAL Id: hal-01326376 \\ https://hal-ensta-bretagne.archives-ouvertes.fr/hal-01326376}

Submitted on 3 Jun 2016

HAL is a multi-disciplinary open access archive for the deposit and dissemination of scientific research documents, whether they are published or not. The documents may come from teaching and research institutions in France or abroad, or from public or private research centers.
L'archive ouverte pluridisciplinaire HAL, est destinée au dépôt et à la diffusion de documents scientifiques de niveau recherche, publiés ou non, émanant des établissements d'enseignement et de recherche français ou étrangers, des laboratoires publics ou privés. 


\section{Experimental Measurement of Time Difference Of Arrival}

\author{
Hugo Seuté, Cyrille Enderli, \\ Jean-François Grandin \\ Thales Airborne Systems \\ 2 Avenue Gay Lussac, 78990 Elancourt, FRANCE \\ email: \{hugo.seute, cyrille-jean.enderli, \\ jean-francois.grandin\} @ fr.thalesgroup.com
}

\begin{abstract}
In this paper is described an experimental passive localization system based on SDR (Software Defined Radio) components. This system is designed to measure Time Differences of Arrival (TDOA) of radar pulses between two platforms. For a TDOA system, time error between the two receivers must be kept very low, which requires a very accurate way to synchronize the time bases. In this purpose, a custom offline synchronization method is proposed. The overall performances of the system are analyzed. In a small scale outdoor experiment, it has been shown to perform TDOA measurements accurately. The performances measured during this experiment are then extrapolated to a more realistic electronic warfare scenario.
\end{abstract}

Index Terms-TDOA; Synchronization; Passive Localization; Electronic Support Measures; Software Defined Radio;

\section{INTRODUCTION}

In several domains such as Electronic Warfare (EW), acoustics, or more recently lightning impact localization or sensor networks, the problem of passive localization based on measures of TDOA has been a central topic in many publications [1-4].

TDOA-based systems use the differential delay of a signal received on distant platforms in order to compute an estimate of the position of the radiating source. In two dimensions, with two platforms, one is capable to position the emitter on an isoTDOA curve, which is a hyperbola. By adding a third platform, the source can be localized at the intersection of the two hyperbolas [2]. Since the measurements depend on the time on several remote platforms, several clocks are needed. All clocks have imperfections which make them drift, yet a single time base must be maintained all along the measurement time, hence the need to synchronize the two devices [3].

This article focuses on an Electronic Support Measure (ESM) system designed for TDOA localization in a short-base biplatform EW context. This is a challenging scenario for a distributed system because the measured time differences are small $(100-1.000 \mathrm{~ns})$ thus the error on TDOA measurements must be kept well under these values in order to achieve good localization performance [3]. This is why a custom synchronization protocol has been developed to ensure a low synchronization error. In the scope of this article we will try to

\author{
Ali Khenchaf, Jean-Christophe Cexus \\ Lab-STICC, UMR CNRS 6285, ENSTA Bretagne \\ 2 Rue François Verny, 29806 Brest, FRANCE \\ email: \{ali.khenchaf, jean-christophe.cexus \} \\ @ensta-bretagne.fr
}

determine what performance in terms of global TDOA error and localization error we can expect from a real system.

The paper is organized as follows: Section II presents the global architecture of the system; Section III introduces the custom synchronization and data acquisition protocol; Section IV describes the processing used to compute TDOA measurements; Section V presents the experimental results which are discussed and extrapolated to a real-life scenario; finally, concluding remarks and perspectives are exposed in Section VI.

\section{ARCHITECTURE OF THE SYSTEM}

The system which was developed for this experimental study is based on two Software Defined Radio (SDR) platforms based on Ettus USRP B210 (Receiver \#1) and B200 (Receiver \#2) cards, linked to a laptop computer to record the data. This setup was chosen because SDR platforms allow quick development and experimentation tasks, moreover USRP $\mathrm{B} 2 \mathrm{XX}$ devices work on a wide band of radiofrequencies (from $70 \mathrm{MHz}$ to $6 \mathrm{GHz}$ ) [5] and are available off-the-shelf and relatively inexpensive.

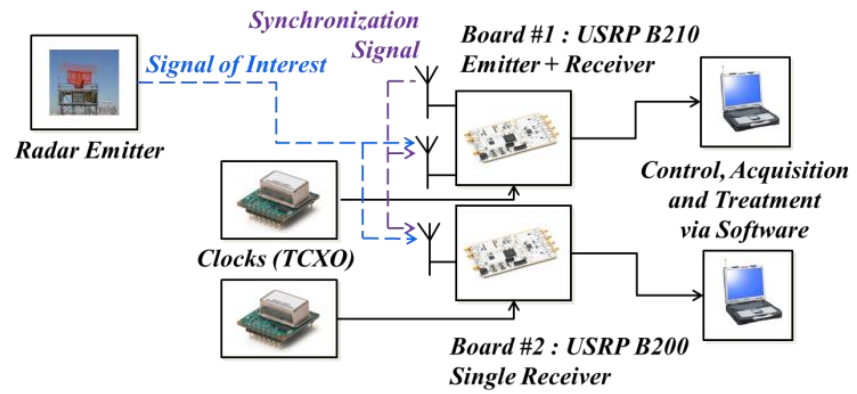

Figure 1. Architecture of the developed system

This system is illustrated in Figure 1. The two platforms work independently, there is no wire connection between them, so a clock on each platform and a mean of synchronization are required in order to measure TDOA. The chosen clocks are Temperature Compensated X (crystal) Oscillators (TCXO) because they have good short-term stability properties [6]. Synchronization is achieved by emitting a signal from platform 1 (see section III). 
After being tuned to the desired frequency, the two direct conversion quadrature receivers start receiving the synchronization signal and the signal of interest (in our case a radar pulse train). They send I/Q data to their attached laptop computer connected via USB 3 [5]. On each platform a piece of software retrieves the I/Q samples and records them into a timestamped file on the computer's SSD (Solid State Drive). These files are then collected and processed offline.

\section{SYNCHRONIZATION PROTOCOL}

\section{A. Overview of the process}

In order to have an accurate way to correct time error between the two receivers, a synchronization protocol has been developed, based on the periodic emission of a sync signal. The emitter of this signal is actually collocated with receiver \#1, inside the B210 card. Its center frequency is set close to the frequency of the signal of interest so that it fits within the receivers' band and the two signals are recorded together. This sync signal is used for two things:

- When it is detected (via a simple arbitrary threshold on the energy in signal's band), the receivers record samples into a file. This avoids to record permanently and to have a huge amount of data to process in the following steps.

- The complex envelopes of the sync signals recorded by the two receivers are cross-correlated to accurately estimate the delay difference between them, as described later (see Fig. 2).

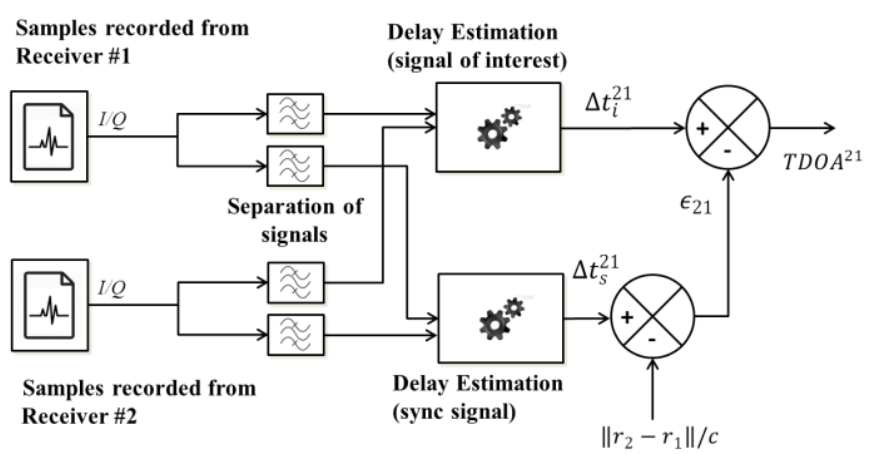

Figure 2. Diagram of the offline processing

\section{B. Choice of a synchronization signal}

There are different requirements on this signal:

- When cross-correlation is computed from the envelope of the sync signals contained in the two files, the crosscorrelation peak must be unambiguous: there must be a single peak which occurs inside an interval of possible delays. Indeed, if the sync signal is periodic with a short period - shorter than the maximum time offset expected between the two records - there will be several cross-correlation peaks that could correspond to a consistent delay estimation.

- This cross-correlation peak also needs to be narrow to improve the accuracy of delay estimation. In [4] is stated that the standard deviation of the time offset estimation is proportional to $1 / \beta$, where $\beta$ is the "rms frequency" of the cross-correlated signals. Hence the synchronization signal needs to have high frequency components to provide accurate delay estimation.

A signal complying with these requirements has been chosen arbitrarily (its envelope is represented on Figure 3). It is composed of a carrier modulated in amplitude by a low and a high frequency signal (satisfying respectively the first and second requirement):

$$
s(t)=\sin \left(2 \pi f_{1} t\right) \sin \left(2 \pi f_{2} t\right) e^{j 2 \pi f_{L O} t} .
$$

where $f_{1}$ and $f_{2}$ are the frequencies of the modulated signals and $f_{L O}$ is the carrier frequency.

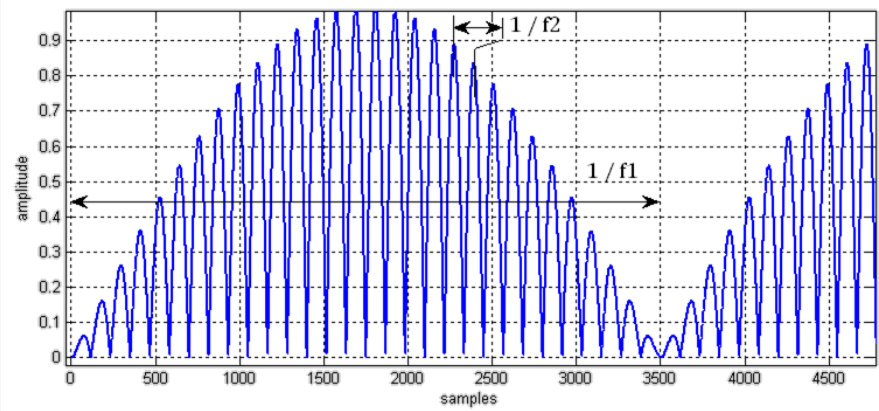

Figure 3. A representation of the envelope of the sync signal with $f_{1}=1 \mathrm{kHz}$ and $f_{2}=30 \mathrm{kHz}$, sampled every $T=143 \mathrm{~ns}$

The settings used during the following experiments are grouped on Table 1.

\begin{tabular}{|c|c|l|}
\hline $\boldsymbol{P}$. & Value & Description \\
\hline$T_{S}$ & $3 \mathrm{~s}$ & Sync signal period of emission \\
\hline$T$ & $143 \mathrm{~ns}$ & Sampling period \\
\hline$D_{r}$ & $200 \mathrm{~ms}$ & $\begin{array}{l}\text { Duration of the record of the sync } \\
\text { signal }\end{array}$ \\
\hline$f_{1}$ & $1 \mathrm{kHz}$ & $\begin{array}{l}\text { Frequency of the first modulation } \\
\text { of the sync signal }\end{array}$ \\
\hline$f_{2}$ & $3 \mathrm{MHz}$ & $\begin{array}{l}\text { Frequency of the second } \\
\text { modulation of the sync signal }\end{array}$ \\
\hline$f_{L O}$ & $5 \mathrm{GHz}$ & $\begin{array}{l}\text { Nominal frequency of the local } \\
\text { oscillators on the receivers and } \\
\text { emitter }\end{array}$ \\
\hline
\end{tabular}

Table 1. Values of the parameters of interest

\section{Delay estimation}

After being filtered out from the recorded data, the synchronization signals received on the two platforms go through several steps in order to estimate the delay between them. These steps are depicted on Figure 4. 


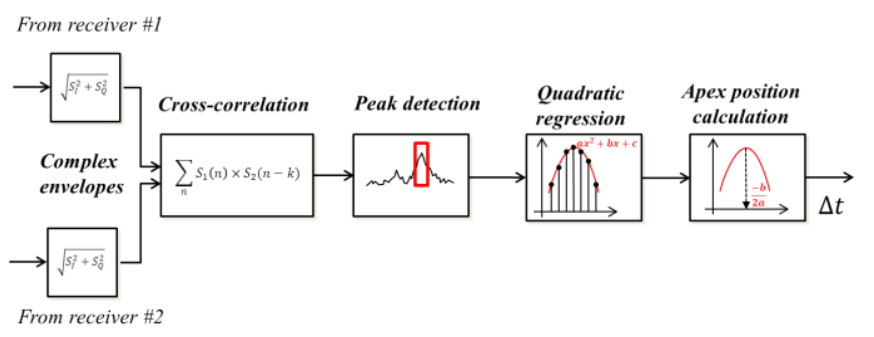

Figure 4. Diagram of the delay estimation process

First, $S_{1}$ and $S_{2}$ the envelopes of the sync signal received on platform 1 and 2 are cross-correlated [2][4]:

$$
R_{S_{1} S_{2}}(k)=\sum_{n} S_{1}(n) S_{2}(n-k)
$$

Where $k$ is the delay (in samples) for which the crosscorrelation is computed. In our case, $R_{S_{1} S_{2}}(k)$ is computed for $k \in\left[\frac{-1}{2 T f_{1}}, \frac{1}{2 T f_{1}}\right]$. A representation of the cross-correlation output for the sync signal is given on Figure $6 \mathrm{a}$.

Then the main peak of the cross-correlation values is identified and a quadratic regression is applied to the points around the apex (in our case the regression is applied to 10 points). This regression calculates the polynomial $a_{1} x^{2}+$ $a_{2} x+a_{3}$ that fits the peak. The final estimate of the delay is the abscissa of the parabola, given by:

$$
\Delta t_{s}^{21}=-\frac{a_{2}}{2 a_{1}} \text {. }
$$

This value corresponds to the delay of the sync signal received by receiver 2 relative to the same signal received by receiver 1 . We introduce $r_{1}=\left[\begin{array}{lll}x_{1} & y_{1} & z_{1}\end{array}\right]^{T}, r_{2}=\left[\begin{array}{lll}x_{2} & y_{2} & z_{2}\end{array}\right]^{T}$ the coordinates of the antennas of the two platforms. It is now possible to compute the time correction offset $\epsilon_{21}$ to subtract to the time base of receiver 2 so that the two time references are coherent:

$$
\epsilon_{21}=\Delta t_{s}^{21}-\left\|r_{2}-r_{1}\right\| / c .
$$

Where $c$ is the speed of light.

\section{TDOA MEASUREMENT}

Using the exact same method that we used in Section III to compute the delay in reception of the sync signal $\Delta t_{s}^{21}$, we can compute the delay in reception of the signal of interest $\Delta t_{i}^{21}$.

Here the treatment is applied to an external signal, which is in the case of EW a signal emitted by a radar system. A typical radar signal, consisting in a periodic series of short pulses (Fig. $5)$, is generated during the experiments.

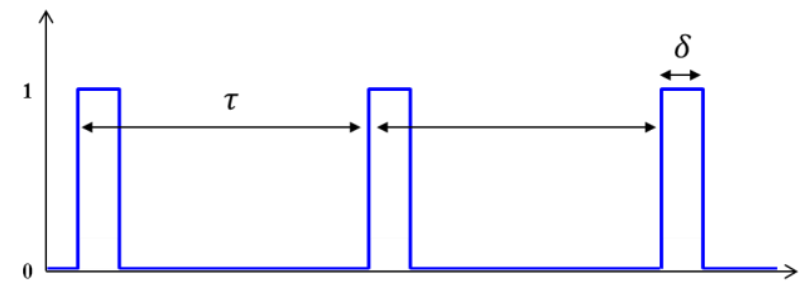

Figure 5. Envelope of the signal of interest, with a PRI (Pulse Repetition Interval) $\tau$ and pulse duration $\delta$
The cross-correlation of the envelope of the signal of interest is shown on Figure 6b.

The final (corrected) TDOA measurement is obtained by subtracting the time offset calculated in III to the value of $\Delta t_{i}^{21}$ :

$$
T D O A=\Delta t_{i}^{21}-\epsilon_{21} .
$$

With the processing steps described in Sections III and IV, one is now capable of computing synchronized TDOA measurements from raw signals coming from two independent receivers.

\section{RESULTS AND ANALYSIS}

An experiment has been set up in an outdoor environment (the soccer field of the ENSTA Bretagne campus). A microwave signal generator linked to a horn antenna was configured to emit a pulse train in order to simulate a radar emission. The characteristics of this signal are given in Table 2. The disposition of the different elements is given in Figure 8. The distances were measured using a laser telemeter. The error on the distance measures is comprised between 1 and $2 \mathrm{~mm}$ [7]. It is considered to be sufficiently low to be neglected on the following steps.

\begin{tabular}{|l|c|l|}
\hline $\boldsymbol{P}$. & Value & Description \\
\hline$f_{c}$ & $5.002 \mathrm{GHz}$ & Carrier frequency \\
\hline$\tau$ & $1 \mathrm{~ms}$ & Pulse Repetition Interval (PRI) \\
\hline$\delta$ & $80 \mu \mathrm{s}$ & Duration of a pulse \\
\hline \multicolumn{3}{|c|}{ Table 2. Values of the parameters of interest }
\end{tabular}

Figure 6 represents the cross-correlation computed from the actual measurements of the signal (after filtering).

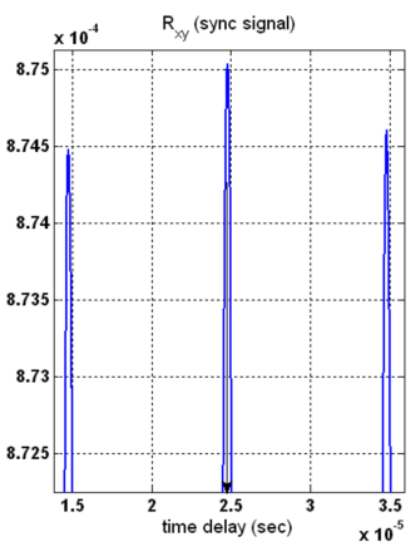

a.

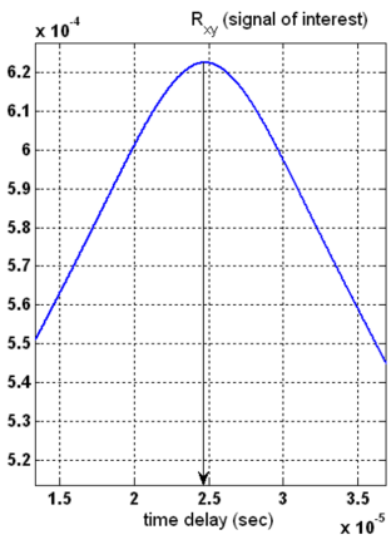

b.
Figure 6. Cross-correlation of the complex envelope of the sync signal (a.) and the generated pulse train (b.)

Corrected TDOA values are computed as described in sections III and IV for 60 measurements, spaced by $T_{s}$. In Figure 7, the real difference of path of the signal (measured with a laser telemeter) is subtracted to the measured TDOAs in order to evaluate their error. 


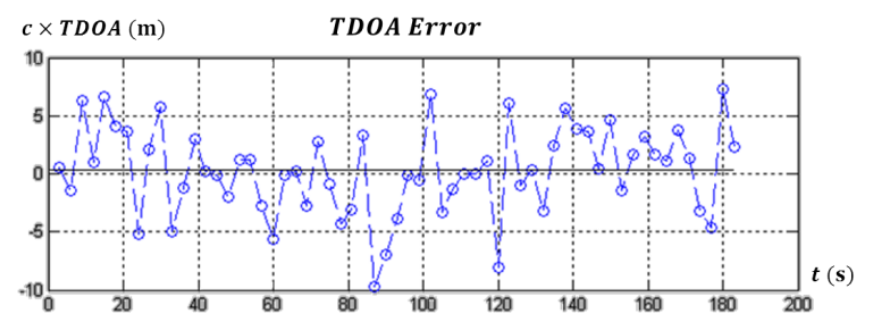

Figure 7. Errors of the TDOA measurements. One point represents one TDOA measurement.

For a single measurement we have $c \cdot \sigma_{T D O A}=4 \mathrm{~m}\left(\sigma_{T D O A}=\right.$ $13 \mathrm{~ns})$. After integration over the 60 measurements, the TDOA error is $30 \mathrm{~cm}(1 \mathrm{~ns})$. In figure 8 is plotted the iso-TDOA hyperbola [2] from the integrated value of TDOA we found, as well as iso-error curves.

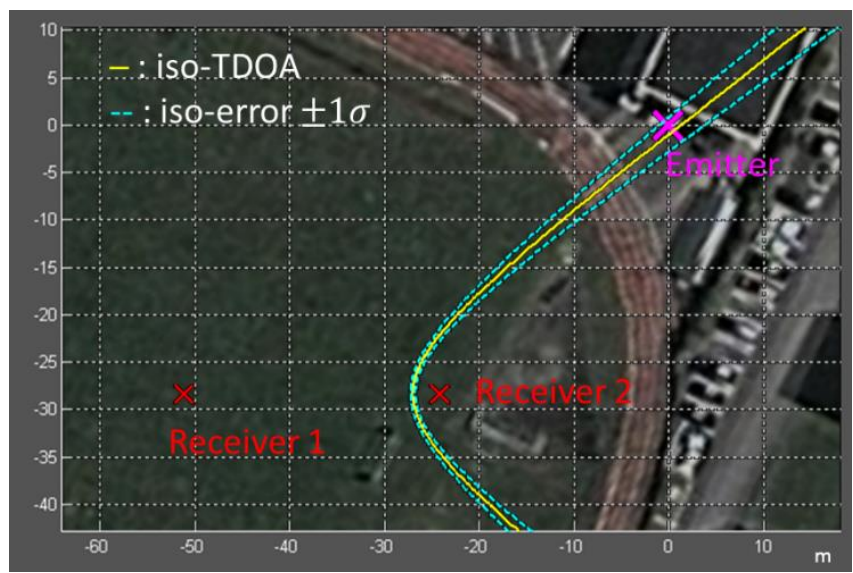

Figure 8. Representation of the geometry of the experiment, with iso-TDOA and iso-error curves

The results obtained in this experiment can be extrapolated to an operational scenario. For example, in electronic warfare we can consider the following case: a patrol of two airborne platforms (fighters or UAVs) are intercepting the lobe of a surveillance radar device far away from them [3]. The distance between aircrafts is $a=1 \mathrm{~km}$ and they both are approximately at a distance of $d=100 \mathrm{~km}$ from the radar. Since $d \gg a$ it is possible to consider a TDOA measurement as an angular measurement [2], then the standard deviation of TDOA corresponds to an angular standard deviation given by:

$$
\sigma_{\theta}=\frac{c \cdot \sigma_{T D O A}}{a \sin \theta}
$$

where $\theta$ is the Angle Of Arrival (AOA) relative to the base orientation.
Considering the same TDOA error variance as in the experimental measurements $\left(\sigma_{T D O A}=13 \mathrm{~ns}\right)$, a $1-\mathrm{km}$ long base and an AOA of $45^{\circ}$, using (3) we find an angular error of standard deviation $\sigma_{\theta}=0.3^{\circ}$ which is quite good, considering that many ESMs are capable of measuring an AOA only with an accuracy of a few degrees [8].

\section{CONCLUSION AND PERSPECTIVES}

To conclude, in this article we have detailed a way to measure TDOA values from two independent platforms using SDR devices. A custom synchronization protocol was presented and its accuracy was studied. Eventually, we investigated the overall standard deviation of TDOA measurement using this setup. Its performances appeared to be quite good, proving that it is possible to have a functional TDOA-based ESM system using affordable off-the-shelf components. Nevertheless, the actual system is not yet capable of full online autonomous operation in a realistic context; it requires that many parameters are tuned manually, such as the gain of the receivers. Work is currently in progress to address these shortcomings.

\section{REFERENCES}

[1] Poisel, R.; "Electronic warfare target location methods". Artech House, 2012

[2] Musicki, D.; Koch, W., "Geolocation using TDOA and FDOA measurements," in Information Fusion, 2008 11th International Conference on , pp.1-8, June 30 2008-July 3 2008

[3] Seute, H.; Grandin, J.-F.; Enderli, C.; Khenchaf, A.; Cexus, J.-C., "Why synchronization is a key issue in modern Electronic Support Measures," in Radar Symposium (IRS), 2015 16th International, pp.794-799, 24-26 June 2015.

[4] Stein, S., "Algorithms for ambiguity function processing," in Acoustics, Speech and Signal Processing, IEEE Transactions on, vol.29, no.3, pp.588-599, Jun 1981.

[5] "USRP specification sheet", Ettus Research; online: http://www.ettus.com/content/files/b200-

b210_spec_sheet.pdf, 2016

[6] Seute, H.; Grandin, J.-F.; Enderli, C.; Khenchaf, A.; Cexus, J.-C., " Experimental Analysis of Time Deviation on a Passive Localization System", submitted to IGARSS 2016.

[7] "Leica Disto D2 User Manual", Leica; online: http://lasers.leica-geosystems.com/fr/sites/lasers.leicageosystems.com.fr/files/product_documents/user_manual _d2_en.pdf, 2016.

[8] Boyd, C. C.; "International Electronic Countermeasures Handbook", Horizon House, Artech House, 2004. 